\title{
A case study of smoke detector assembly work method intervention and evaluation
}

\author{
Chuan Sun ${ }^{\mathrm{a}, *}$, Bryan Buchholz ${ }^{\mathrm{a}}$ and Jeff Thomas ${ }^{\mathrm{b}}$ \\ ${ }^{a}$ Department of Work Environment, University of Massachusetts Lowell, Kitson 200, One University Ave, Lowell, \\ MA, 01854, USA. \\ ${ }^{b}$ UTC Fire \& Security in Maine, 129 N Main St.Pittsfield,ME,04967,USA
}

\begin{abstract}
The study aimed to evaluate a work method intervention in a smoke detector assembly line. High hand gripping forces and extreme wrist postures were found in the smoke detector assembly line at UTC Fire \& Security Company in Maine. A fixture was introduced to replace the old assembly method. Electromyography (EMG) and electrogoniometry were used to measure the workers' hand gripping force and wrist motions with both the old and new assembly methods. Results show both hand gripping forces and wrist postures improved significantly with the new method.
\end{abstract}

Keywords: Ergonomics, EMG, Extreme wrist postures, High hand forces

*Corresponding author. 1-978 9302486 E-mail: chuan_sun@student.uml.edu. 


\section{Background}

UTC Fire \& Security Company in Maine (UTCFS) invited the Department of Work Environment at the University of Massachusetts Lowell (UMass Lowell) to help identify and reduce ergonomic hazards in the smoke detector final assembly stage. The intervention aimed to reduce the highly repetitive motion and high hand gripping force hazards during the smoke detectors' chamber and housing installations.

Cumulative trauma disorders (CTD) have been found to affect as much as $25 \%$ of the work force in some jobs and are a major cause of lost time from work [1]. CTD have been shown to be precipitated by the use of particular hand and wrist postures while performing repetitive works or with forceful exertions [2]. Silverstein et al. [3] mentioned that the hand jobs loadings between $14.5 \pm 6.2 \mathrm{~kg}$ was considered as high forces. The intervention project used $20.7 \mathrm{~kg}(202 \mathrm{~N})$ as the upper limit for grip force.

\section{Idea development and new work method}

\subsection{Brain storming}

A brain storming activity was performed together by the UMass Lowell students and the UTCFS technical group. Creative suggestions were raised including using machines and fixtures for automated alignment and assembly of the housings to replace the delicate, forceful and repetitive hand motions. Due to cost and timing issues, the project finally focused on using available material in the plant to minimize the ergonomic hazards.

\subsection{Idea realization}

In the old assembly method, the final assembly procedure mainly composed of two steps: 1) Align and fix the inner chamber onto the smoke detector's base. 2) Align and fasten the outer cover onto the base (Figure 1, Step C). Workers usually accomplished the two steps by pressing or twisting with the fingers, and both of the two steps contain stressful pressing operations and extreme wrist postures. The intervention aimed to: 1) Reduce extreme wrist extension/flexion 2) Reduce the high hand force in the pressing operation.

In the new assembly method (Figure 2), a manual pressing fixture was used as the prototype machine. A "cover holder" and "base supporter" were installed on the fixture. The "cover holder" is a round rubber pad that was used to keep the outer cover from moving. The "base supporter" is a rotational fixture that is used to hold the smoke detector's base. To finish the assembly work, workers need to place the aligned parts onto the "base supporter", rotate the right knob down vertically to make the rubber pad clamp onto the outer chamber. At the same time, the left hand will rotate the yellow knob beside the "base supporter". Figure 1 shows the old assembly method and Figure 2 shows the new assembly method.

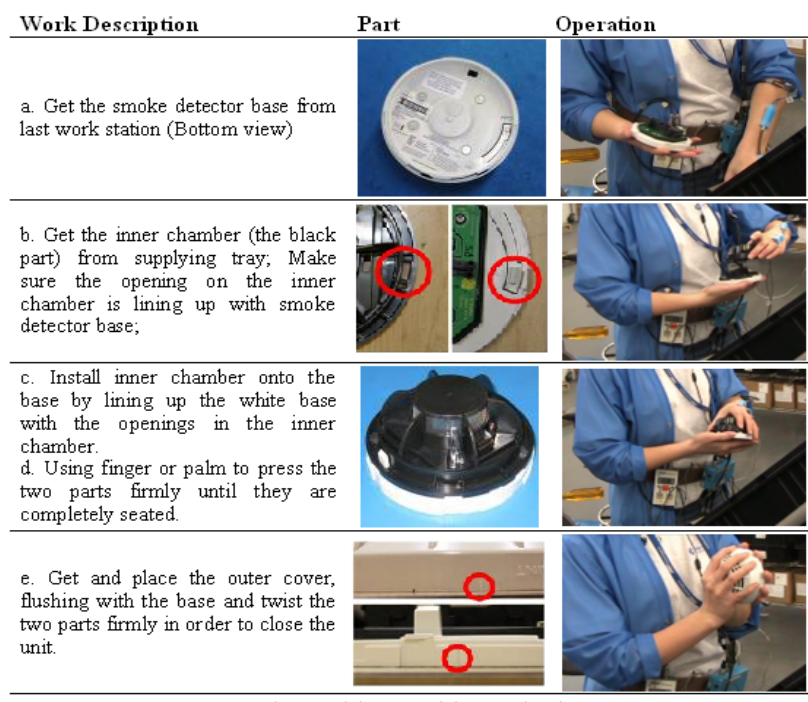

Fig 1 Old assembly method

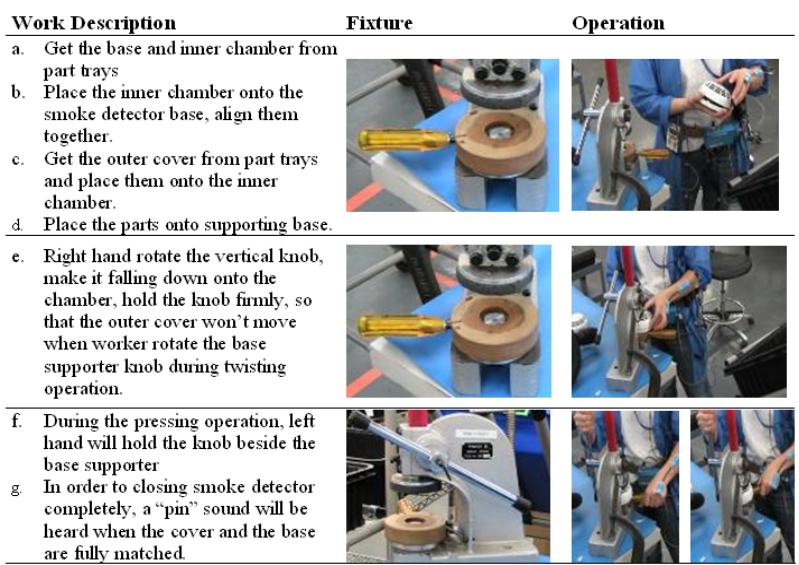

Fig 2 New assembly method

\section{Method}

Electromyography (EMG) was used to estimate the muscle forces for both hands and a twin axis electrogoniometer (Penny and Giles, G65) was used to 
measure wrist motions on right hand. Data were originally collected at the UTCFS facility, but because of difficulty with the EMG signal due to electromagnetic interference, simulations were also performed at the Occupational Biomechanics Lab at UMass Lowell. Assembly task simulations were performed in both places with both old and new methods.

One female subject at UTCFS and one male subject from the Occupational Biomechanics Lab participated in the experiments. Before the experiment, researcher read an informed consent form to the subjects, and the subjects signed the form to indicate the consent to participate. Disposable EMG electrodes were placed close to Flexor Digitorum Superficialis (FDS) on the subject's forearm and were fixed with medical adhesive tapes. A portable data logger (Tattletale $5 \mathrm{~F}$ ) was attached to the waist belt on the subject. At UTCFS, the subject performed 10 cycles of assembly work using both the new and old methods. Before and after the assembly work, subjects were asked to use both hands to perform maximum gripping forces on a dynamometer, while force value and EMG signal were simultaneously recorded. A hand forces to EMG linear regression model was established. During the task simulation, the whole process was recorded with a static video camera, no personal identifying information was recorded. The EMG data were transformed into force $(\mathrm{N})$ using the linear regression and were analyzed together with video records.

In the Occupational Biomechanical Lab, the assembly work simulation was similar to the experiment at UTCFS. The only difference in the Lab was that the subject completed 6 to 7 cycles of assembly work, and there was a short break ( 1 to 2 seconds) between every assembly cycle.

The simulation tasks at the two locations were recorded with a video camera. The averaged assembly cycle times in the old and new assembly methods at the two places were read from the video graph during the data analysis.

\section{Results}

The dependent variables in this study were hand gripping force and right wrist flexion/extension during the assembly simulation work at the two locations.

\subsection{Hand gripping force evaluation at UTCFS}

Figure 3 shows one of the ten cycles (from time point 66 second to 82 second) of assembly simulation at UTCFS. As is displayed, the right hand peak twisting force at the time point of 79 s reached $400 \mathrm{~N}$ in the old assembly method. Figure 4 (cycle time from 203 second to 220 second) shows that both right and left hand peak twisting forces at the time point of $217 \mathrm{~s}$ reached $200 \mathrm{~N}$ in the new assembly method.

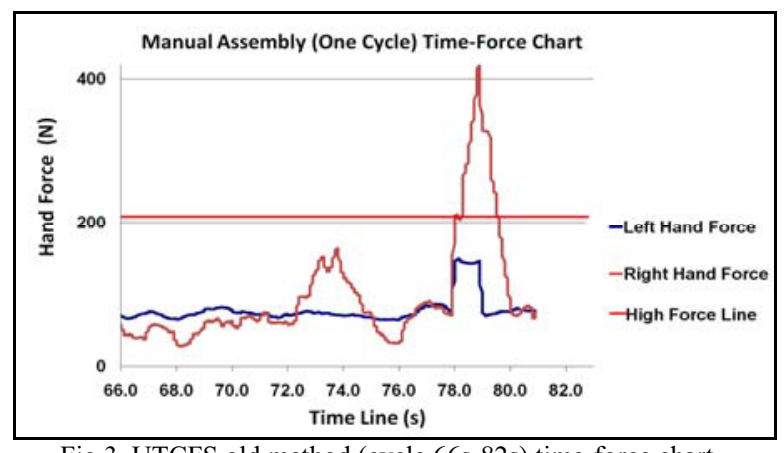

Fig 3, UTCFS old method (cycle 66s-82s) time-force chart

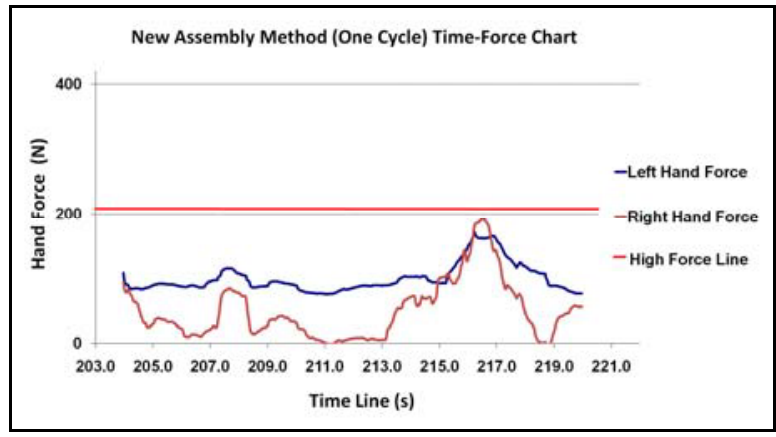

Fig 4, UTCFS new method (cycle203s-220s) time-force chart

\subsection{Hand force evaluation at biomechanics lab}

In the Biomechanics Lab experiment, Figure 5 and Figure 6 show 10 cycles of assembly simulation with old and new assembly method respectively. In both figures, when subject's right hand were not pressing and twisting, the hand forces were below the high force level $(202 N)$. Compare the force value in the old method (Figure 5) from time point 60 seconds to 70 seconds with the force value in the new method (Figure 6) from time point 60 seconds to 65 seconds, the hand force peak value lasted for a longer time than it is in the new method. 


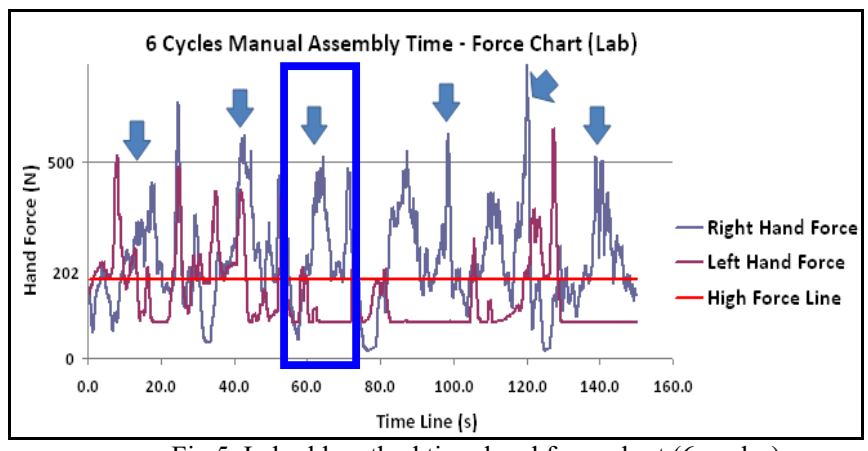

Fig 5, Lab old method time-hand force chart (6 cycles)

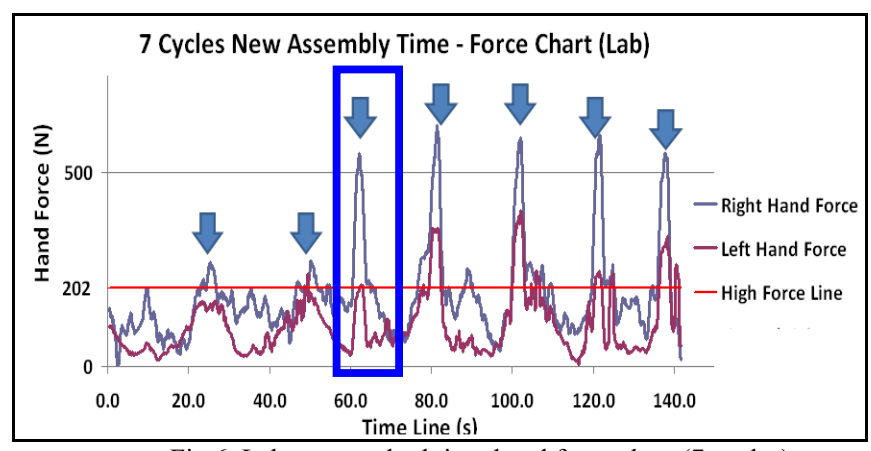

Fig 6, Lab new method time-hand force chart (7 cycles)

\subsection{Hand wrist motion evaluation at UTCFS}

With the old method (Figure 7) shows that during the 10 cycles of assembly simulation at UTCFS, the number of times that right hand wrist extension exceeding 45 degrees was greater than 9 . With the new method (Figure 8) shows that during the 10 cycles of assembly simulation, the number of times that right hand wrist extension exceeding 45 degrees was 2 .

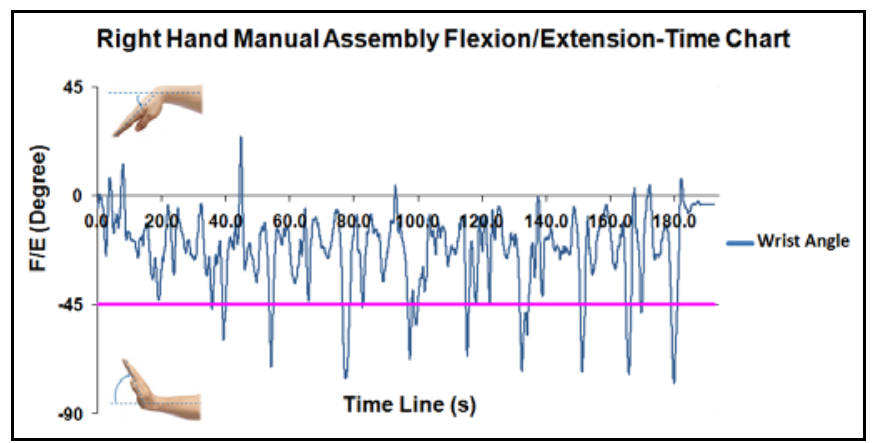

Fig 7, UTCFS Right hand old method F/E-time chart (10 cycles)

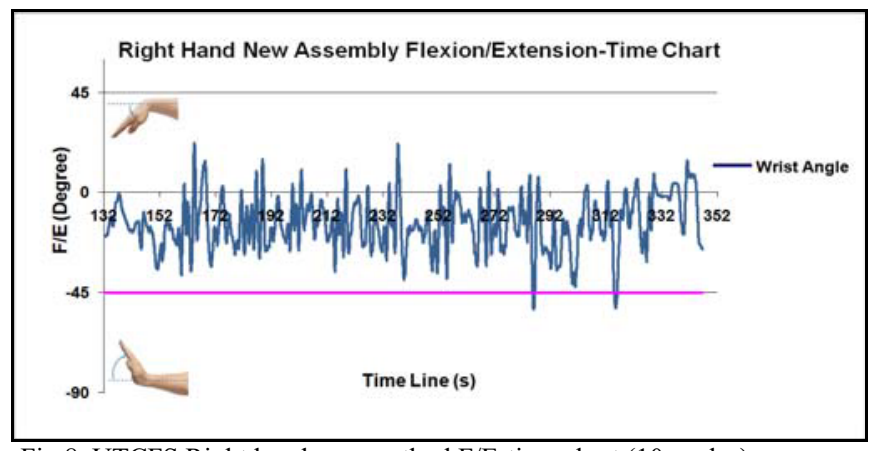

Fig 8, UTCFS Right hand new method F/E-time chart (10 cycles)

\subsection{Hand wrist motion evaluation at biomechanics lab}

In the Biomechanical Lab, Figure 9 and Figure 10 show that the wrist flexion and extension on right hand were below 45 degrees in both the old and new methods.

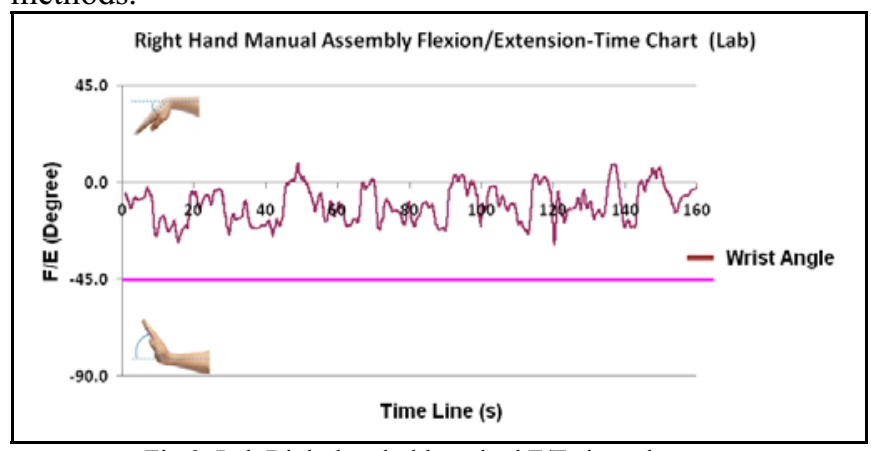

Fig 9, Lab Right hand old method F/E-time chart

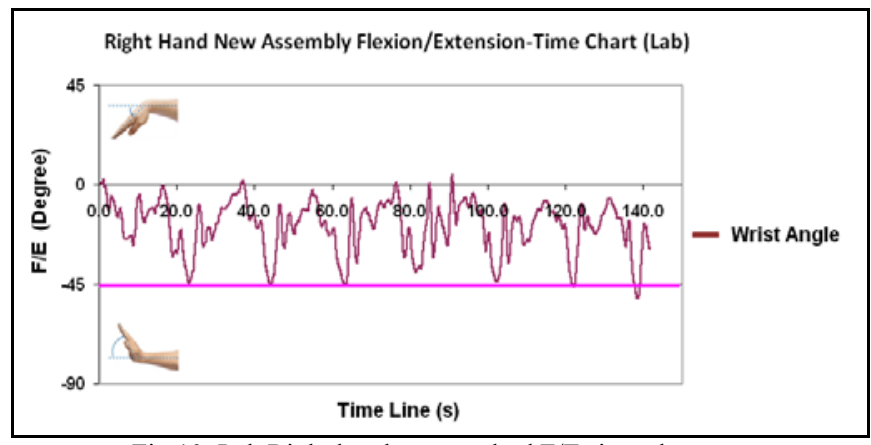

Fig 10, Lab Right hand new method F/E-time chart

\subsection{Assembly cycle time study in both methods}

In UTCFS, the subject assembled 10 pieces of work with the old and new method. The subject in Biomechanics Lab assembled 7 pieces of works with the same methods. Figure 11 shows the different as- 
sembly cycle times with the different methods in the two locations.

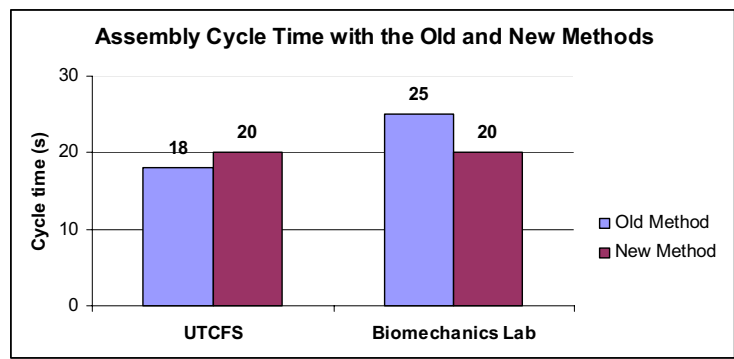

Fig 11, Assembly cycle time with different methods

\section{Discussion}

In the experiments at UTCFS, Figure 3 and 4 shows decreased right hand peak gripping force with the new method in one of the ten assembly cycles. In the Biomechanics Lab's experiment, although some of the peak gripping forces is higher than the high force level $(202 \mathrm{~N})$, the duration of peak hand gripping force has been shortened. Such observations may ascribe to the reduced finger press and adjustment motions in the new assembly method.

Figure 7 and 8 shows that the right hand wrist extension decreased in the new assembly method at UTCFS. In Figure 9 and 10, the right hand wrist extension increased a little bit in the new method, but still not exceeding 45 degrees. Possible explanations could be: As shown in Figure 12, with the old assembly method in the lab, the subject's arms were placed a distance from the body, and the hand wrist kept in a neutral position. In the old method in UTCFS, subject placed arms close to the body as showed in Figure 13. A short distance between forearm and body allowed the subject to have better visual control, but such practice may introduce extreme wrist extension.

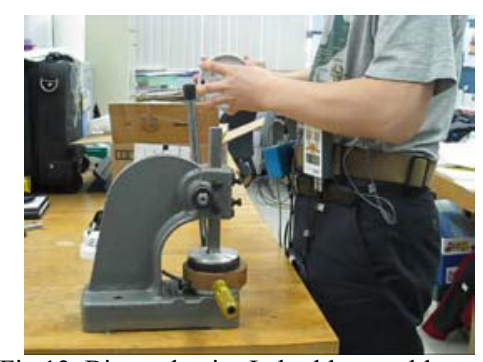

Fig 12, Biomechanics Lab old assembly method

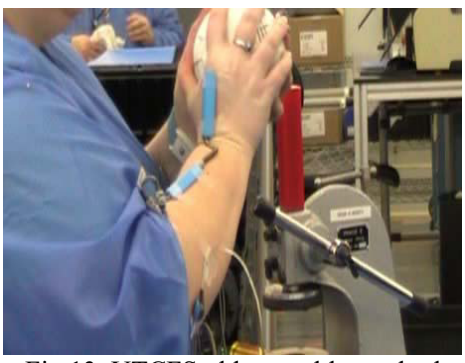

Fig 13, UTCFS old assembly method

As shown in Figure 11, the average cycle time in the new method was higher than the one in the old method in UTCFS. But in the Biomechanics Lab, the cycle time is shorter in the new method. A plausible explanation could be: 1) subject in UTCFS got used to work with the old method and thus it cost less time.2) subject in the Lab neither familiar with the old method nor familiar with the new method, it cost the subject more time in the old method than in UTCFS. However, the average assembly cycle time in the Biomechanics Lab is shorter in the new method.

Qualitative feedback from the users who are using the new method in UTCFS shows: 1) that the new method cause less stress on the hands and wrists, and that it makes the pressing and twisting job easier to be accomplished than in the old method. 2) Complaints from the users focused on the functionality of the fixture. Feedback suggested improving the pad design, in that the pad was reported to cause some damages to the LED lights on the outer chamber during the pressing operations.

\section{Limitation}

Although the study showed improvement with the new assembly method, there is still some limitations of concern.

\subsection{Limitation one: small sampling size}

During the site visit and lab experiment, only two subjects' assembly simulations were successfully measured. Doubts may be cast on whether the experiment subjects represent the other workers? In fact, subject's working habit did make differences. In the old assembly method, subject who was recorded by the video camera showed a few differences in assembly approach. Possible explanation could be that workers didn't follow the assembly guidelines. 


\subsection{Limitation two: difference between experiment settings and real assembly scenario}

The experimental settings may be different from the real scenario in terms of the following aspects: 1 ) in the real production, the material logistics may be different in terms of location and flow from the settings in the experiment. 2) time spent on the final assembly stage in the real production may be longer than it was in the experiment in that there could be some re-work of mis-assembled detectors in the real scenario.

\subsection{Limitation three: systematic measurement error}

The experiment used many electrical and signal processing devices, which might bring about measurement error and noise that are produced in the equipments per se. The noise caused by the equipment is difficult to be avoided. Such barriers can be avoided with use of an advanced portable EMG data recording system.

\section{Recommendation}

\subsection{Recommendation one}

As shown in Figure 14, future fixture design should set the length ratio between shorter arm and longer arm of the pressing (vertical) knob as $1: 3$, in addition, it is recommended to replace the yellow knob with an automatic rotation machine that rotate the supporter base as soon as the pressing (vertical) knob is pressed. Such design will save workers' energy (larger force moment) and allow the vertical knob to automatically return to the original point after releasing it.

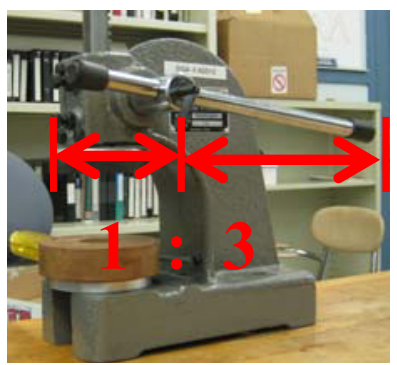

Fig 14, Recommended Design

\subsection{Recommendation two}

The ultimate goal of the ergonomic project was to help reduce the ergonomic hazards and increase the productivity. At the same time, the product's quality and production reliability shall not be influenced due to the changes. Therefore, it is strongly recommended to upgrade the current fixtures with an advanced assembly machine and logistic system that could satisfy a higher production output in the long run.

\section{References}

[1] Armstrong, TJ, Foulke, JA, Joseph, BS, and Goldstein, SA. Investigation of cumulative trauma disorders in a poultry processing plant. American Industrial Hygiene Association 43 2/82, 1992.

[2] Armstrong, TJ, Radwin, RG and Hansen, DJ. Repetitive trauma disorders: Job evaluation and design. Human Factors 28(3), 325-336, 1986.

[3] Silverstein, B. A., Fine, L. J., \& Armstrong, T. J. Occupational factors and carpal tunnel syndrome. American Journal of Industrial Medicine 11(3), 343-358, 1987 